\title{
MATHEMATICS IN THE SOCIAL SCIENCES: REFLECTIONS ON THE THEORY OF SOCIAL CHOICE AND WELFARE
}

\author{
MARIANO GIAQUINTA E HYKEL HOSNI
}

\section{Mathematics in the SoCial SCiEnCES}

Mathematical activity can be seen as originating in response to two kinds of stimuli. For definiteness, and not very imaginatively, let us refer to them as internal and external stimuli, respectively. The kind of work is motivated by internal stimuli focusses on problems which come, as it were, in mathematical form, and whose solutions are subject solely to the success criteria of the particular area (or areas) of mathematics within which the problem has arisen. A substantial part of the so-called "pure mathematics" falls squarely within this category, though we hasten to add that we find the "pure" vs. "applied" terminological and conceptual divide more misleading than helpful. The second kind of stimulus to mathematical work comes from the attempt at solving problems which originate outside the area(s) of mathematics in which they are solved. The domains in which the question arise can be extremely varied and may relate both to applications (e.g. in engineering, medicine, etc.) and to theoretical scientific questions. Among the latter one can distinguish the sort of mathematics which is motivated by the natural sciences - mainly physics, chemistry and biology - from the kind of mathematics which develops in response to the questions posed by the social sciences.

The distinction we are putting forward, rough as it may be, is motivated by the following observation. Within the natural sciences, the phenomenon which constitutes the object of scientific investigation is completely characterised by its mathematical description. So, for example, Euler's equations for the dynamics of physical systems, rather than providing a "mathematical model" of something which isn't mathematical, do constitue in themselves the description of the phenomenon of interest. Contrast this with the social sciences where the mathematical formalisation of its key concepts can often be seen as the culmination of a historically complex process of abstraction

Date: 28 luglio 2015. 
which, in some cases, may lead to stripping out important aspects of the phenomenon of interest. A particularly relevant case in point, on which more below, is provided by the hypotheses on the agents' rationality which can be found in virtually every emanation of mathematical economics, and in particular, in general equilibrium theory. Assuming that individuals are omniscient and logically infallible is something methodologically very different from considering frictionless motion. To assume that consumers are omniscient is to model something other than the individuals which act in the "real" market. The ensuing loss of predictive power of a theory based on such modelling assumption becomes then all too obvious and calls for a careful methodological analysis.

Before moving on with the formulation of a specific question concerning the role of mathematics in the social sciences, we must remind readers that the tradition which ultimately culminated in modern mathematical economics takes the relation between natural and social sciences to be quite different from the rendering we have just put forward. Indeed for a large portion of the twentieth century the justification for the mathematical modelling in the social sciences consistently pivoted on physical analogies. Vilfredo Pareto (1848-1923), to make a very notable example, noted how the controversial notion of homo economicus should be regarded as the pure economics counterpart of the abstraction with which rational mechanics reduces bodies to points. Such abstractions, says Pareto in [8] are "similar" and are motivated by "similar needs". Interestingly, Pareto adds a footnote to the word "similar" referring to he paper in which Vito Volterra (1860-1940) introduces his Prey-predator model.

Similar considerations are put forward by von Neumann e Morgenstern in [10] in connection with the introduction of the concept of utility. This concept, to which we now refer as "von-Neumann and Morgestern utility" provides a key part of the language of contemporary mathematical economics. We could list many more notable examples, certainly not confining ourselves to the past century. Let us just observe, as an aside, that analogy, in problems of quantitative finance, has been replaced with a fusion of economics and finance, giving rise to the field known as econophysics.

Going back to our main theme, the purpose of this note is to articulate some necessarily rough and sketchy reflections on mathematical modelling in the social sciences. The focus of our attention will be restricted to the central problem of the theory of social choice and welfare. The development will be informal and space limitations will force us to gloss over a number of important questions. We refer interested readers to consult [3] for the 
mathematical precise details and its bibliography for the bigger picture.

\section{Mathematical ECONOMiCs AND the ILlusion of the EFFicient} SELF-ORGANISATION OF SOCIETY

Problem What is the best way to allocate economic resources within a given society?

Building on the common roots of social choice and welfare problems we will refer to the above simply as to the problem of social choice. This is undoubtedly one of the key problems in economic theory and its history can be traced back easily to the political arithmetics of revolutionary France. Given its centrality, it should not come as a surprise that the main political ideologies of our time can sometimes be told apart according to the point of view they take on the formulation of the problem of social choice, and consequently, on the relative attempts at providing a solution to it.

According to a view which is promoted mainly by the right-wing libertarians, the solution to the problem of social choice is contained in a mathematical argument which revolves around the fundamental theorem of welfare economics. Informally, this view takes social welfare to be the product of the interaction - within a free market - of rational individuals who are motivated solely by the satisfaction of their (economic) goals. It goes without saying that the relevance and importance of the theorem with respect to the problem of social choice depend centrally on the interpretation and the justification of the mathematical modelling which ultimately leads to its formulation. A central modelling hypothesis in this respect falls under the general term of methodological individualism. The idea, roughly, consists in assuming that the analysis of society as a whole can be reduced (without loss of explanatory power) to the analysis if its ultimate components, namely individual rational agents. This rather abstract concept is one of the most significant items of the Enlightenment's Nachlass to the social sciences, and indeed a concept which plays a central role in a number of distinct areas. The key idea is that rational agents - which in various cases will take the guise of consumers, voters or players - are characterised by their own preferences over the socio-economic alternatives of interest. This identification allows us to put the multifaceted and subtle concept of "rationality" in the rigorous, albeit restrictive, terms of the logical coherence of preferences. As a major consequence of this framework, rational decision can be identified with maximising behaviour in suitably specified contexts. Hence, with a handful of seemingly quite innocent conceptual steps, the concept of rationality, both individual and collective, can be formalised in such a way as 
to make the problem of social choice amenable to mathematical treatment and, possibly, solution.

Before illustrating informally the fundamental theorem of welfare economics, it is worth pausing for a second to note the consonance between conceptual framing of social choice provided by methodological individualism and the vision put forward by G.W. Leibniz concerning the algorithmic nature of ideal reasoning. For one of the inventors of modern logic any controversy between two philosophers about which he writes:

For it would suffice for them to get their pencils, sit down each to his abacus and (urged by a friend, if they please so ) say to one another: Calculemus!

Indeed we will be investigating the conditions which are necessary and sufficient for a society's rational preferences, i.e. those representing the will and well-being of the collectivity, to be obtained as the value of a suitable function which takes as argument the preferences of the rational individuals which belong to that society.

If such a formal mechanism existed, it could well put forward a serious claim for defining rigorously a central aspect of the concept of democracy. Desirable as this may be however, such a functional aggregation mechanism cannot exist. This is indeed the conclusion of a series of results which, starting with an observation of the Marquis de Condorcet, culminated in modern times in the well-known Arrow Theorem [1]. Roughly the theorem asserts the following:

Theorem 1. Consider a society of at least two rational agents who must express their preferences on at least three alternatives. It is impossible to aggregate logico-mathematically the individual preferences into a collective preference which while satisfying the principle of Unanimity does not coincide with the dictatorship of one individual.

To provide a rigorous formulation of this theorem, which will also help us appreciate some basics of mathematical modelling, we need a little notation and some definitions. Suppose $S$ is a society consisting of $n$ individuals, each of whom is identified with a preference ordering, that is to say a relation $\succcurlyeq_{i}$ defined on the set $\mathcal{X}=\{X, Y, \ldots\}$ of social alternatives. We assume that $\succcurlyeq_{i}$ satisfies the following:

Ordering: $\succcurlyeq_{i}$ satisfies

- reflexivity: $X \succcurlyeq_{i} X \quad \forall X$

- transitivity: if $X \succcurlyeq_{i} Y$ and $Y \succcurlyeq_{i} Z$ then $X \succcurlyeq_{i} Z$; 
- completeness: for each pair $(X, Y)$ exactly one of the following holds: either $X \succcurlyeq_{i} Y$ or $Y \succcurlyeq_{i} X$.

Suitable considerations based on methodological individualism lead us to define an aggregation rule which takes individual preferences $\succcurlyeq_{i}$ with $i=$ $1, \ldots, n$ as argument, and which gives as value the social preference $\succcurlyeq_{S}$. In other words we are interested in rules satisfying the condition of

Functionality: For each pair of social alternatives in $\mathcal{X}^{2}, R$ is of the form

$$
\succcurlyeq_{S}=R\left(\succcurlyeq_{1}, \ldots, \succcurlyeq_{n}\right)
$$

Note that Ordering and Functionality correspond essentially to requiring that the solution to the problem of social choice be of a logico-mathematical nature. It is not hard to see, in this regard, that the condition of Ordering which defines rationality both at the individual and social level, is rooted in the logical - and hence purely formal - concept of coherence. Functionality, on the other hand, is closely reminiscent of the compositionality (or truthfunctionality) of the classical propositional calculus, and this latter provides a language in which the whole problem of social choice, and indeed Arrow's own theorem, can be fruitfully reformulated. (Again, we refer the interested reader to [3] for more details.)

In addition to the requirements of coherence - i.e. of formal correctness - that we have just introduced, in order for the aggregation rule $R$ to be materially adequate with respect to the intended interpretation of "social choice", further constraints of economic and political nature must be introduced. In other words we want to make sure that the mathematical model under construction is a model wich bears direct relevance to social choice, rather than being merely an abstract (albeit interesting) exercise. Here is where the distinction between natural and social sciences discussed in the previous section becomes central. The kind of constraints to be imposed on $R$ will be clearly external to mathematics, and yet they will be quite far from providing an exhaustive description of the phenomenon of interest (in contrast to the example of motion recalled above). Mathematical modelling in the social sciences requires its own methodology.

Since a direct formalisation of the concept of "social choice" appears too daunting to even try, a rather natural alternative route consists in the exclusion of those situations which appears to be blatantly inconsistent with the most basic intuitions related to the concept of social choice. In other words our aim is to justify the desirable properties of $R$ by arguing that 
they prevent us from incurring in a process of aggregation which satisfies properties that we do not want $R$ to satisfy

The first undesirable such property is quite clearly that $R$ identifies social choice with the unilateral preference of one member of the society. That is to say we impose the axiom of

Non-dictatorship: For no $i=1 \ldots, n$,

$$
R\left(\succcurlyeq_{1}, \ldots, \succcurlyeq_{n}\right)=\succcurlyeq_{i}
$$

A rule which did not output as social choice the preference unanimously held by all members of the society would likewise be clearly inadequate. To rule this situation out we require that $R$ satisfies the axiom of

Unanimity: If $X \succcurlyeq_{i} Y$ with $i=1 \ldots, n$ then $X \succcurlyeq_{S} Y$.

Finally a constraint which originates directly from the methodological individualism assumption. It is undesirable that, the aggregation of individual preferences on alternatives $X$ and $Y$, depended on something other than the individual preferences on $X$ and $Y$. More precisely we impose the axiom of

Independence: Suppose that $\succcurlyeq_{i}$ and $\succsim_{i}$ are two families of orderings with $i=1, \ldots, n$, and suppose that both satisfy the condition of Functionality $\succcurlyeq_{S}:=R\left(\succcurlyeq_{1}, \ldots, \succcurlyeq_{m}\right)$ and $\succsim_{S}:=R\left(\succsim_{1}, \ldots, \succsim_{m}\right)$. Then

$$
X \succcurlyeq_{i} Y \Leftrightarrow X \succsim_{i} Y, \quad \forall i
$$

implies

$$
X \succcurlyeq_{S} Y \Leftrightarrow X \succsim_{S} Y
$$

Intuitively, the condition says that if two distinct preference profiles coincide on social alternatives $X$ and $Y$, then we do not want $R$ to produce distinct aggregations of the two profiles. This condition, which in various guises occupies centre stage in the mathematics of social sciences, is often referred to as Independence of irrelevant alternatives. Indeed any aggregation rule $R$ satisfying int, dismisses as irrelevant the information contained in the preference profile $\succcurlyeq_{i} \mathrm{e} \succsim_{i}$ on alternatives other than $X$ and $Y$.

Arrow's theorem establish the mutual incompatibility of the conditions of Ordering Functionality, Non-dictatorship, Unanimity and Independence. A particularly enlightening formulation of the theorem is as follows:

Theorem 2 (Arrow, 1950). Suppose Ordering and Functionality hold, and suppose that $R$ satisfies Non-dictatorship and Unanimity. Then $R$ cannot satisfy Independence. 
Put in this way this much celebrated result sheds interesting light on the impossibility of addressing the problem of social choice in a purely logicomathematica way: if we have good reasons to impose the remaining conditions, we must necessarily give up Independence. And with this, we must abandon the requirement to the effect that the aggregation of individual preferences - in close analogy with the Calculemus - is sufficient to determine the preference of the whole society without any external intervention

Theorem 2 therefore provides us both with a diagnosis and with a suggestion as to how the impossibility of the logico-mathematica aggregation may be overcome within an Arrovian framework. As illustrated in the next section, this happens by weakening increasingly the methodological individualism hypothesis, and in particular its instantiation based on the condition of Independence.

\section{The Fundamental theOREM OF Welfare ECONOMiCS}

Arrow's theorem provides an admirable illustration of the contribution brought by mathematics to the formulation of specific problems in the social sciences. As the informal discussion of the previous section suggests, the role of mathematical analysis is mainly to ensure the internal coherence of the model as a consequence of the rigorous and careful scrutiny of its fundamental assumptions. As pointed out by Bruno De Finetti [2]

Used critically and accompanied by a reflection on the concrete features and problems of the portion of reality which it aims at modelling, mathematics is a $[\ldots]$ constructive analytical tool because it is hard to tame and often leads to destruction: those contradictions, inconsistencies, discontinuities, asymmetries which could otherwise go unnoticed to those who limited themselves to imagine mental or verbal descriptions, are clearly brought to light by to those who consider the mathematical model[...]

The contradiction brought to light by the Arrow theorem is about the mutual incompatibility of hypotheses which, at first sight, have nothing incompatible about them. To the contrary both appear to be natural and compelling, especially to those who believe in the market's ability to self-regulate and to promote social welfare through purely individual incentives.

We therefore must replace some of the hypotheses which give rise to Arrow's framework with others capable of leading us to a solution to the problem 
of social choice. As anticipated, we will focus mainly on the assumption of methodological individualism. Before getting into any of the details, we must provide some further background on the Arrow-Debreu model of abstract economy.

Consider a specific instance of the general problem of social choice, that is to say the allocation of $l$ commodities (which we assume are available in a total quantity $\Omega$ ) to the $n$ individuals which belong to society $S$. In this context, Arrow's theorem tells us that any allocation satisfying Ordering, Functionality, Non-dictatorship and Unanimity will necessarily violate Independence - a logico-mathematical solution cannot exist unless we allow in the evaluation an external element with respect to the individual preferences on the social alternatives. It turns out that a kind of comparison between individual preferences allows to make an important step forward towards the solution. Such a comparison - which is not permitted within the Arrovian framework - is made mathematically possible by a set of results which lie at the heart of mathematical economics. Those results identify the necessary and sufficient conditions under which the following are proved to be logically equivalent: (i) the representation of the individual agents' preferences by means of orderings and (ii) their representation by means of suitable utility functions. $^{1}$

This allows us to make quite some progress. For an individual in society can now be identified with a suitable real-valued utility function, and very naturally, we may press on to take say, the function

$$
u_{S}=u_{1}+\ldots+u_{n}
$$

to represent the collective preference of society. Now we can reiterate the representation of preferences, that is given two social alternatives $X$ and $Y$

$$
X \succcurlyeq_{S} Y \text {, if and only if } u_{S}(X) \geq u_{S}(Y),
$$

means that society prefers $X$ to $Y$ just when the social utility of $Y$ is not greater than that of $X{ }^{2}$ In general this representation is obtained up to a choice of parameters, in the sense that we can always write aggregate utility

\footnotetext{
${ }^{1}$ Indeed, under rather mild conditions, every ordering can be represented (non-uniquely) by suitable utility functions.

${ }^{2}$ As anticipated above, the concept of utility is central in virtually all mathematical models in the social sciences. And yet cavalier interpretations often lead to misunderstanding which we do not have the space to prevent here. To this end we refer the reader to [3] and in particular to Chapters 3 and 4.
} 
functions as

$$
u_{S}^{\alpha}=\alpha_{1} u_{1}+\ldots+\alpha_{n} u_{n}, \quad \alpha_{i} \geq 0 \quad \forall i, \quad \sum_{1}^{n} \alpha_{i}=1 .
$$

The continuity of the utility functions which we take into consideration guarantees, via Weierstrass's Theorem, that for each $\alpha$ the function $u_{S}^{\alpha}$ attains a maximum $X^{\alpha}$ in the domain of social alternatives (which are now interpreted as possible allocations) $\mathcal{X}$. By the equivalence (1), this provides us with enough background to define a fundamental concept of mathematical as well as political economics, namely Pareto efficiency. Before giving its definition however, recall that given a preference ordering $\succcurlyeq \subseteq \mathcal{X}^{2}$ (i.e. a reflexive, transitive and total relation over the social alternatives) it is completely standard to define:

- $\sim$ (the indifference relation) by letting

$$
X \sim Y \Leftrightarrow X \succcurlyeq Y \text { and } Y \succcurlyeq X
$$

- $\succ$ (the asymmetric part of $\succsim)$ by letting

$$
X \succ Y \Leftrightarrow X \succcurlyeq Y \text { but not }(X \sim Y) .
$$

Definition 1. Say that $X$ is a weak Pareto maximum (optimum) if the following is satisfied:

$$
\nexists Y \in \mathcal{X} \text { such that } Y \succ_{i} X \quad \forall i .
$$

Say that $Y$ is a strict Pareto maximum (optimum) if the following is satisfied:

$$
\nexists Y \in \mathcal{X} \text { such that } Y \succcurlyeq_{i} X \quad \forall i \text { and } Y \succ_{j} X \quad \text { for some } j \text {. }
$$

Intuitively, a social alternative is efficient, in the sense of being a Pareto maximum or optimum, if it increases the utility of at least one member of society without making anyone else worse off. Under the hypothesis that individual and social utility functions describe completely ${ }^{3}$ what is relevant to the individual and to society as a whole - clearly not a innocent assumption - an efficient allocation in the sense of Pareto cannot be improved upon. A moment's reflection is sufficient however to see how the criterion of Pareto efficiency may lead to clearly unfair outcomes as it rules out as inefficient any kind of redistribution of the economic resources within society. Once again we are forced to refer the interested reader to [3] for further ethicopolitical reflections on Pareto efficiency and urge those with an interest in the topic to consult [9] which also contains a very detailed list of references.

\footnotetext{
${ }^{3}$ Think again about Euler's equation for the motion of physical systems.
} 
Let us resume then our quest for a mathematical solution to the problem of social choice by illustrating the key contribution of Pareto efficiency. Under suitable technical conditions on the individual preference orderings, one can prove that for each social alternative $Y \in \mathcal{X}$, there exists a strict Pareto maximum $X \in \mathcal{X}$ which is unanimously preferred to $Y \in \mathcal{X}$, that is one such that $X \succcurlyeq_{i} Y$, with $i=1, \ldots, n$. Under the hypothesis to the effect that Pareto efficiency singles out "the best" allocations of economic resources to society, the above guarantees the existence of a solution to the problem of social choice in which a form of interpersonal preference comparisons is now possible. This form of comparison, it is worth emphasising, means giving up some of the strictness of the Independence assumption which leads to the Arrow theorem. It is easy to guess however that this solution is far from being unique and the Paretian framework within which the solution is found, provides us with no further formal criterion for the selection of one among the many Pareto optima. Again, for the identification of a concrete solution to the problem of social choice we need further external intervention.

To this end we enrich the Paretian model with the concept of private property. In addition to their own preferences over the social alternatives, each member of society $i$ is now endowed with a commodity bundle $\omega^{i} \in \mathcal{X}$. We assume that the totality of commodities, denoted by $\Omega=\omega^{1}+\ldots+\omega^{n}$ has been distributed among the $n$ individuals.

The introduction of private property gives us an opportunity to say something more about the hypothesis of methodological individualism. In particular we are assuming that $i$ 's individual preferences (and hence utilities) are purely self-centered in the sense that $u_{i}$ depends only on the commodity bundle $\omega^{i}$ (and only on that.) This leads to a private property economy, arguably the simplest context in which the notion of a market makes full sense. Indeed a commodity is such insofar as there is someone who finds it desirable. Therefore the introduction of private property gives us some elbow room to reformulate the problem of social choice as the problem of exchange of private commodities. In this enriched context, the interpersonal comparison among individuals which emerged in the Paretian framework gains further importance.

The idea is roughly as follows. Each individual possesses a commodity bundle whose worth (measured in utilities) is assumed to be additive. A concept which arises naturally in this situation is that of a coalition to which the individual agents participate to the extent that the coalition promotes their self-centered interests. The mathematical analysis of coalitions within a private property market has many interesting consequences, but this is not 
the best place to discuss them. We just limit ourselves to mentioning that, in a suitably specified context, we can associate to each private property economy a cooperative game and investigate the problems arising in the former by applying the solution concepts of the latter. ${ }^{4}$ Note in passing that this provides an enlightening illustration of the role of mathematics in the construction of economic models. Indeed a significant portion of the theory of cooperative games (or coalitional games) developed as a consequence of an external stimulus, to reiterate the terminology used in the first section, namely by the problem of identifying the conditions under which the core of private property economy is non-empty. This, in turn, gave rise to a series of results, mainly of geometric and topological nature, which litte, if anything at all, have to do with the initial economic interpretation. To give the reader a feel for the situation we are alluding to, let us just mention that the well known Shapley theorem - a key result in cooperative games - implies the theorems of Knaster, Kuratowski and Mazurkiewicz, which imply Brower's fixed-point theorem, which in turn (a posteriori) turns out to be equivalent to Shapley's own theorem.

Let's go back to private property economies. A characteristic feature of coalitions is that they can block those Pareto optima which are considered not to be in the coalition's best interests. Such are those allocations which, if realised, would lead to a smaller value (in utilities) than the sum of the utilities which the individuals could secure independently of the coalition. Against this background, and under suitable technical conditions, in addition to the assumption to the effect that each individual may belong, to a certain degree, to multiple coalitions (referred to as fuzzy coalitions), one can prove the existence of prices which are associated to the commodity bundles.

An important observation is in order. In the abstract economies (with private property) which constitute the background to this result, prices emerge as a purely formal consequence of the economic interaction among self-centered agents. In other words prices are "chosen" by the market. Thus if one could prove that prices obtained in this way do indeed promote social welfare, one could reasonably claim to possess a strong argument in favour of (a suitably adapted version of) methodological individualism, indeed one which is pretty much in line with the free-market narrative. To some, this is precisely the contribution of the fundamental theorem of welfare economics. Before stating and discussing its relevance informally, we need to introduce a final set of concepts.

\footnotetext{
${ }^{4}$ Full details are available from [3].
} 
The analysis of coalitions led to the determination of prices in terms of (the coefficients associated to) the allocations which belong to the core $\mathcal{W}$ of fuzzy coalitions. As a preliminary observation note that, under suitable conditions, one can prove that fuzzy coalitions do not block all efficient allocations in the sense of Pareto. That is to say $\mathcal{W}$ is non-empty. The language and results of game theory allow us to characterise $\mathcal{W}$ rigorously in terms of Walras equilibria. To do so suppose that a particular price system is given for the abstract economy of interest. By making some extra assumptions on the self-centered rationality of economic agents, namely that they always prefer more wealth to less - note that the price system allows us to measure wealth interpersonally - we can prove that any allocation in the fuzzy core is logically equivalent to an allocation which is in equilibrium with respect to a suitable price system.

The concept of equilibrium featuring here is essentially the one which developed in the context of neoclassical economics, and which was formulated rigorously for the first time by Leon Walras (1834-1910). In essence, it amounts to the requirement that the demand and supply commodities must be balanced. Building on this, one can show that every equilibrium in the sense of Walras is efficient in the sense of Pareto. If one also assumes that a suitable redistribution of the initial commodities is possible, the converse can also be proved, namely that Pareto optima are themselves Walras equilibria. That gives us, in essence, the statement of the Fundamental Theorem of Welfare Economics:

Theorem 3 (Hotelling-Allais-Arrow-Debreu). Suppose all the initial resources have been allocated to rational, self-centered agents (who possess a certain quantity of each commodity) then

(1) every Walras equilibrium is a Pareto optimum and

(2) conversely, every Pareto optimum is a Walras equilibrium with respect to a price system and a redistribution of the commodities.

Let us recap the story so far, and let us do so while keeping in mind that, contrary to what may be suggested by our highly informal and simplified exposition, the historical development of the methods and concepts which culminated in Theorem 3 has been far from linear. We started by asking whether one could argue mathematically for or against a certain way of making public decisions, that is to say decisions involving social welfare. We took it for granted that our (of the present writers, probably of the readers, and reasonably of a substantial part of the population) cultural background leads us naturally to give preference to the point of view which, for lack of a better word, we have been calling democratic. In other words we would 
like decisions about social welfare to depend suitably (!) on the will of the individuals which constitute society. Motivated by such ideas, we briefly touched upon the most significant steps leading to the construction of the Arrovian framework and to the ensuing impossibility of devising a logicomathematical method of aggregating individual preferences into the will of society. However, by weakening the hypothesis of methodological individualism a new path unfolded before us, this time based on Pareto efficiency. Trying to refine the selection of Pareto optima through the formation of coalitions of individuals motivated uniquely by the maximisation of their own personal utility, and owing to the introduction of private property, we arrived at the determination of a price system for which the market is to be considered as the only responsible. Theorem 3 tells us that market-set prices are efficient in the sense of Pareto, and conversely, that Pareto optima lead to equilibrium prices. Since this result generalises to the economy with production, the question naturally arises as to wether this proves, as a practical corollary of the Fundamental Theorem, that the best way to promote social welfare is indeed to incentivise the pursuit of individual welfare within a free, competitive market. Put otherwise, have we proved the existence and the intrinsic efficiency of the invisible hand put forward by the founding father of Modern Economics, Adam Smith?

According to the point of view which we articulated in this note, the answer can be affirmative only to the extent that we are willing to accept all the assumptions and hypotheses used in the construction of the mathematical model as a complete characterisation (in the sense illustrated in the first section) of the phenomena of interest. However there are several reasons to doubt that strong arguments can be put forward to that effect, except possibly for some "local" versions. Let us illustrate the idea starting with with some relatively obvious considerations on the nature of economic agents.

- Economic omniscience: A necessary condition for the derivation of Theorem 3 states that each individual consumer knows the price of each commodity, at every fixed place and time. Clearly if this hypothesis is ever to be satisfied, it can only be in very special circumstances, relative that is to a given commodity bundle at one specific place, etc.

- Logical omniscience: Contrary to the hypotheses concerning ideal rationality (as they are captured by the identification of rational decision with the maximisation of subjective utility) consumers often violate the logical criteria of rationality - and, as observed by behavioural economists, they do so very predictably. As Jonathan Swift put it men aren't quite rational animals, but rather animals which 
are capable of being rational.

- Perfect liquidity: Likewise necessary is the assumption to the effect that each consumer is always in a position to buy or sell the desired quantities of commodities at market price. This is again an utterly unrealistic hypothesis which is virtually impossible to satisfy in realworld economies. There cannot be, for a start, any guarantee that commodities will be available on the market when desired.

Therefore, pace Pareto, irrationality, i.e. the deviation from the logical criteria of coherence and maximisation, isn't quite friction. To neglect it, is to strip away a characteristic feature of the object of scientific inquiry for which the model is being constructed.

But even if homo economicus gave way in mathematical economics to a more earthly homo heuristicus - as envisaged by the Bounded Rationality research project ${ }^{5}$ - why should we ever believe that the result of economic activity in free markets really possesses the characteristic properties of Walras equilibria? The proof of the existence, within the model, of a price system in equilibrium certainly does not amount the proof that those prices which are observed in a real-world economy coincide with those balancing supply and demand. Again let us illustrate informally the reasons why caution, if not scepticism, is in order when evaluating such an identification.

- Black Box: The concept of Walras equilibrium doesn't provide us with any information as to the actual mechanisms governing markets. It rather refers us to decidedly unrealistic devices, like the Walrasian Auctioneer, which essentially amounts to the following. An auctioneer announces a price $p$; if this turns out not be the equilibrium price, he then chooses another price $q$ with $q_{k}<p_{k}$, in case of positive demand excess in $p_{k}$, and $q_{k}>p_{k}$, in case of negative excess demand, until the equilibrium is reached. Whilst mathematically this corresponds to a powerful tool, it is clearly unrealistic to think of equilibrium as arising at the same time in all markets, and for all consumers.

- Experimental testability: Last, but certainly not least: can we test the predictions of general equilibrium theory experimentally? The question is far from trivial. Suffice it to recall here the contribution of Hugo Sonnenschein who, during the 1970s, raised the question in the following terms. Is methodological individualism sufficient to provide non trivial, experimentally testable constraints on aggregate

\footnotetext{
${ }^{5}$ See e.g. [4].
} 
excess demand as well as on market demand? A number of specialists (including Rolf Ricardo Mantel, Gérard Debreu, Pierre André Chiappori e Ivar Ekeland) responded negatively, showing how equilibrium theory is generally ill-suited to produce empirically falsifiable predictions. The last word on this intriguing and highly complex issue is however far from having been written.

\section{Conclusions}

Essentially the same arguments which lead us to a form of scepticims concerning the actual consequences, for the problem of social choice, of the Fundamental Theorem of Welfare Economics appear to lead us to a more general form of scepticism, which concerns the relation between mathematical models in the natural and social sciences. Arrow, Debreu, Shapley - not to mention Milnor, von Neumann, Nash, and many more- gave fundamental contributions to the development of economic theory, and by doing so, often gave rise to new research areas in mathematics. Hence there's absolutely no doubt concerning the primary role played by mathematics in the quest for a solution to the fundamental problem of social choice considered in this note.

However, by the line of reasoning that we outlined above(and discussed in more detail in [3]), the role of mathematics is neither hegemomic nor self-sufficient. The mathematical formalisation of the problem of optimal allocation of economic resources within society tells us which paths we must avoid, on pain of inconsistency. But the right-wing libertarians's belief that the mathematical analysis will eliminate all but one solution - the solution according to which social welfare is obtained solely as a consequence of the promotion of individual interests - doesn't seem to be justified at all.

The failure of the paths centered on methodological individualism however should not be taken as a proof of the futility of the initial question. To the contrary, it must be taken as a stimulus to devote greater efforts to developing more adequate methods (and less culturally entrenched ones at that) and ultimately a better theory. Our analysis does in fact suggest the indispensability of a formal framework, but it also suggests its insufficiency to the theoretical understanding of social welfare (and consequently its principled, practical realisation).

Hence is only seems reasonable to insist that the formal results of mathematical economics should always be accompanied with a careful conceptual and experimental analysis of the social phenomena of interest. Those, in turn, should be based on an uninterrupted debate in which the members 
of society oppose, with tolerance, their points of view. In short, a collective deliberation process capable of taking into account the intrinsic cultural diversity and the intrinsic multiplicity of the possible answers. This collective deliberation would ideally aim at improving our understanding of social phenomena and ultimately contribute to a better education for society as a whole. Armed with this renewed understanding, society could then aim at an ever increasing freedom.

The goal is therefore highly ambitious, and the path to it is dotted with tricky concepts and technical difficulties. Daunting as this might sound, not only do we think that this path is viable, but also that it is necessary for us to take it. Thus we leave our final words to Amartya Sen, who argues with the typical elegance, along very similar, though perhaps slightly broader, lines:

The remedy for bad reasoning lies in better reasoning, and it is indeed the job of reasoned scrutiny to move from the former to the latter. It is also possible that in some statements of 'Enlightenment authors' the need for reassessment and caution was not sufficiently emphasized, but it would be hard to derive from that any general indictment of the Enlightenment outlook, and even more, an arraignment of the general role of reason in just behaviour or good social policy. $[9]$

\section{RIFERIMENTI BIBLIOGRAFICI}

[1] K.J. Arrow. Social Choice and Invidivual Values. Jonh Wiley and Sons, second edi edition, 1963.

[2] B. de Finetti. Un matematico e l'economia. Franco Angeli, Milano, 1969.

[3] M. Giaquinta e H. Hosni. Teoria della scelta sociale e Teorema fondamentale dell'economia del benessere: Razionalità, coerenza efficienza ed equità. Edizioni della Normale, 2015.

[4] G. Gigerenzer e H. Brighton. Homo Heuristicus: Why Biased Minds Make Better Inferences. Topics in Cognitive Science, 1(1):107-143, 2009.

[5] S. Leonesi e C. Toffalori. L'arte di uccidere $i$ draghi. Università Bocconi Centro PRISTEM, 2013.

[6] R. Lucchetti. Di duelli, scacchi e dilemmi. Bruno Mondadori Editore, Milano, 2001.

[7] F. Lillo, S. Miccichè, and R. Mantegna. Econofisica: Il contributo dei fisici allo studio dei sistemi economici. Il nuovo Saggiatore, pages 68-81.

[8] V. Pareto. Manuale di Economia Politica. Società editrice libraria, Milano, 1919.

[9] A.K. Sen. The idea of Justice. Harvard University Press, 2009.

[10] J. von Neumann e O. Morgenstern. The Theory of Games and Economic Behavior. Princeton University Press, Princeton, third edition, 1953. 